\title{
Sirtuin 2 knockdown inhibits cell proliferation and RAS/ERK signaling, and promotes cell apoptosis and cell cycle arrest in multiple myeloma
}

\author{
TIANLING DING $^{1}$ and JIE HAO ${ }^{2}$ \\ ${ }^{1}$ Department of Hematology, Huashan Hospital, Fudan University, Shanghai 200040; \\ ${ }^{2}$ Department of Hematology, Shanghai Jing'an District Beizhan Hospital, Shanghai 200070, P.R. China
}

Received January 2, 2020; Accepted November 2, 2020

DOI: $10.3892 / \mathrm{mmr} .2021 .12400$

\begin{abstract}
The present study aimed to explore the regulatory role of sirtuin 2 (SIRT2) in malignant progression of multiple myeloma (MM) and the potential associated signaling pathways. In total, 30 patients with MM and 15 healthy bone marrow donors were enrolled in the current study and their bone marrow samples were collected to isolate the plasma cells. The expression levels of SIRT2 were detected in MM cell lines (KMS-28BM, U266, RPMI-8226 and NCI-H929) and normal plasma cells (collected from healthy bone marrow donors as the control) via reverse transcription-quantitative PCR (RT-qPCR) and western blot analysis. SIRT2 knockdown was established by transfecting two MM cell lines (RPMI-8226 and NCI-H929 cells) with short hairpin RNA-SIRT2 recombinant plasmid; the control group was transfected with a control recombinant plasmid. Subsequently, the effect of SIRT2 knockdown on MM cell proliferation, apoptosis, cell cycle progression and RAS/ERK signaling was investigated via Cell Counting Kit-8, flow cytometry, RT-qPCR and western blot assays, respectively. The mRNA and protein expression levels of SIRT2 were increased in U266 ( $\mathrm{P}<0.001)$, KMS-28BM $(\mathrm{P}<0.001)$, RPMI-8226 $(\mathrm{P}<0.001)$ and NCI-H929 $(\mathrm{P}<0.001)$ cells compared with those in the control cells. In NCI-H929 and RPMI-8226 cells, cell proliferation was decreased $48 \mathrm{~h}(\mathrm{P}<0.05)$ and $72 \mathrm{~h}(\mathrm{P}<0.05)$ after SIRT2 knockdown. Furthermore, the cell apoptotic rate was elevated $48 \mathrm{~h}$ after
\end{abstract}

Correspondence to: Dr Jie Hao, Department of Hematology, Shanghai Jing'an District Beizhan Hospital, 29 Nanxing Road, Shanghai 200070, P.R. China

E-mail: haojunxin@163.com

Abbreviations: MM, multiple myeloma; SIRT2, sirtuin 2; FGFR3, fibroblast growth factor receptor 3; RT-qPCR, reverse transcription-quantitative PCR; p-ERK, phosphorylated-ERK; NAMPT, nicotinamide phosphoribosyltransferase

Key words: apoptosis, cell cycle, cell proliferation, multiple myeloma, RAS/ERK, sirtuin 2
SIRT2 knockdown $(\mathrm{P}<0.01)$. In addition, the percentage of cells at the $G_{0} / G_{1}$ phase was increased $(P<0.01)$, whereas the percentage of cells at the $S$ phase was reduced $(\mathrm{P}<0.01) 48 \mathrm{~h}$ after SIRT2 knockdown. The expression levels of HRAS and phosphorylated-ERK were also reduced $48 \mathrm{~h}$ after SIRT2 knockdown. In conclusion, SIRT2 was highly expressed in MM cell lines, and knockdown of SIRT2 inhibited MM cell proliferation, inactivated the RAS/ERK signaling pathway, and promoted cell apoptosis and cell cycle arrest.

\section{Introduction}

Multiple myeloma (MM) is a heterogeneous disorder of plasma cells that is characterized by uncontrolled proliferation of monoclonal plasma cells, leading to the accumulation of nonfunctional intact immunoglobulins or immunoglobulin chains in the bone marrow (1). As the second most prevalent hematological malignancy, MM accounts for $\sim 1 \%$ of all cancer cases and the current 5-year survival rate is estimated at $\sim 46.6 \%$ worldwide (2). The emergence of several advanced treatments for MM, including autologous stem cell transplants, chemotherapy, targeted drugs and immunomodulatory drugs, has resulted in a marked improvement in the clinical outcomes of patients with MM. However, the majority of patients with MM still suffer from a high risk of relapse and the development of refractory MM, as a result of current therapies (3-5). Therefore, it is essential to explore novel, promising therapeutic targets, which can be applied for the improvement of treatment efficacy in MM.

Sirtuin 2 (SIRT2) is a member of the human sirtuin family, which has a unique $\mathrm{NAD}^{+}$-dependent protein deacetylase activity (6). Emerging evidence has demonstrated that SIRT2 functions as an $\alpha$-tubulin deacetylase, which is implicated in numerous biological processes, including microtubule dynamics, mitotic arrest, cell motility and cell differentiation (7-12). Furthermore, the involvement of SIRT2 in specific pathological processes, such as carcinogenesis, development of leukemia, neurodegeneration and formation of drug resistance has been previously reported (13-16). For example, the role of SIRT2 in regulating aberrant proliferation and survival of myeloid leukemia cells has been reported in former studies. SIRT2 has been shown to promote proliferation 
and survival of acute myeloid leukemia (AML) cells via regulating ERK1/2 signaling and RAS/ERK/JNK/MMP-9 signaling (16-18). In addition, RAS/ERK signaling has been reported to regulate the p85-fibroblast growth factor receptor 3 (FGFR3) interaction in MM, whereas FGFR3 knockdown may inhibit proliferation and promote apoptosis of MM cells $(16,17,19)$. Furthermore, activation of RAS/ERK has been associated with the increased proliferation and survival of MM cells, contributing to the pathogenesis of this disease (20). Based on the aforementioned data, it was hypothesized that SIRT2 might have an important role in the development and progression of MM. However, to the best of our knowledge, the interaction of SIRT2 with MM progression has not been previously reported. Therefore, in the present study, the regulatory effect of SIRT2 knockdown on cell proliferation, induction of apoptosis and regulation of the cell cycle was investigated in MM cells. Moreover, the interaction of the RAS/ERK signaling pathway in MM was examined with regards to the aforementioned processes.

\section{Materials and methods}

Participants. A total of $30 \mathrm{MM}$ bone marrow samples were collected from 30 patients (age range, 28-72 years; sex, 18 males and 12 females) with de novo MM treated at Huashan Hospital (Shanghai, China) or Shanghai Jing'an District Beizhan Hospital (Shanghai, China) between June 2016 and December 2018. All patients had a confirmed diagnosis of de novo symptomatic MM, according to The International Myeloma Working Group criteria of MM (21). The patients were all >18 years old, and had not received radiation or chemotherapy prior to sample collection. The patients were also devoid of other hematological malignancies or solid tumors. In addition, 15 healthy bone marrow samples were collected from 15 bone marrow donors (age range, 29-45 years; sex, 10 males and 5 females) during the same period. The present study was approved by the Institutional Review Board of Shanghai Jing'an District Beizhan Hospital (approval number 2019-021). All participants provided written informed consent for their participation in the study.

Cell culture. Human MM cell lines, KMS-28BM, U266, RPMI-8226 and NCI-H929, were purchased from the American Type Culture Collection. All cells were cultured in RPMI-1640 medium (Gibco; Thermo Fisher Scientific, Inc.) supplemented with 10\% (KMS-28BM, RPMI-8226 and NCI-H929) or 15\% fetal bovine serum (U266) (Gibco; Thermo Fisher Scientific, Inc.) and $1 \%$ penicillin and streptomycin at $37^{\circ} \mathrm{C}$ in a humidified incubator containing $5 \% \mathrm{CO}_{2}$. The plasma cells were isolated from bone marrow mononuclear cells, which were derived from the bone marrow samples of patients with MM and healthy bone marrow donors. Briefly, after collection, the bone marrow samples were processed with gradient density centrifugation $\left(865 \mathrm{x} \mathrm{g}\right.$ at $37^{\circ} \mathrm{C}$ for $20 \mathrm{~min}$ ) for separating the bone marrow mononuclear cells; subsequently, the separated bone marrow mononuclear cells were purified using CD138-coated magnetic beads (Miltenyi Biotec $\mathrm{GmbH}$ ) to obtain plasma cells $(22,23)$. The plasma cells were then stored in liquid nitrogen for further analysis. After incubation at $37^{\circ} \mathrm{C}$ for $24 \mathrm{~h}$, SIRT2 expression in MM cell lines and normal plasma cells (from healthy bone marrow donors that were used as the control group) was determined by RT-qPCR and western blot analysis.

Plasmid transfection. SIRT2 short hairpin RNA (shRNA) and a nonsense shRNA sequence were designed and synthesized by Changchun Changsheng Gene Pharmaceutical Co., Ltd. The sequences for the shRNAs were as follows: shSIRT2 type 1, 5'-GCTAAGCTGGATGAAAGAGAA-3'; shSIRT2 type 2, 5'-GCCAACCATCTGTCACTACTT-3'; shSIRT2 type 3, 5'-CCTGCTCATCAACAAGGAGAA-3'; and shNC, 5'-GCAACAAGATGAAGAGCACCAA-3' (24). The sequences were subsequently cloned into the GenePharma SuperSilencing shRNA ${ }^{\mathrm{TM}}$ vector (pGPU6/RFP/Neo) (Shanghai GenePharma Co., Ltd.) to construct shRNA-SIRT2 (Sh-SIRT2) recombinant plasmid and shRNA-negative control (Sh-NC) recombinant plasmid. Lipofectamine ${ }^{\circledR} 3000$ Transfection Reagent (Invitrogen; Thermo Fisher Scientific, Inc.) was used to transfect the RPMI-8226 and NCI-H929 cells ( $1 \times 10^{5}$ cells/well) with $0.8 \mu \mathrm{g}$ recombinant plasmids at $37^{\circ} \mathrm{C}$ for $24 \mathrm{~h}$, which resulted in the corresponding Sh-SIRT2 and Sh-NC cells. After $24 \mathrm{~h}$, SIRT2 expression in the cells was detected by RT-qPCR and western blot analysis.

Cell proliferation and apoptosis assays. At 0, 24, 48 and $72 \mathrm{~h}$ post-transfection, the proliferation of Sh-SIRT2 and Sh-NC cells was detected using the Cell Counting Kit-8 assay (Dojindo Molecular Technologies, Inc.) in accordance with the manufacturer's instructions. The optical density value of the samples was measured at $450 \mathrm{~nm}$ using the iMark microplate reader (Bio-Rad Laboratories, Inc.). The determination of apoptosis of Sh-SIRT2 and Sh-NC cells was performed $48 \mathrm{~h}$ post-transfection using the Annexin V-fluorescein isothiocyanate Apoptosis Detection kit (Sigma-Aldrich; Merck KGaA), as described previously (17). The apoptotic cells (early + late apoptotic cells; $\mathrm{PI} /$ Annexin $\mathrm{V}^{+}$staining represents early apoptotic cells and $\mathrm{PI}^{+} /$Annexin $\mathrm{V}^{+}$staining represents late apoptotic cells) were analyzed using a CytoFLEX ${ }^{\mathrm{TM}}$ flow cytometer (Beckman Coulter, Inc.) and FlowJo 7.0 software (FlowJo LLC).

Cell cycle analysis. A total of $48 \mathrm{~h}$ post-transfection, the transfected Sh-SIRT2 and Sh-NC cells were harvested by trypsinization and subjected to cell cycle analysis using a BD FACSCalibur flow cytometer (BD Biosciences). The assay was performed as described previously (25). At least 10,000 events were acquired for each sample in order to obtain a measurable signal. The cell percentages at the $\mathrm{G}_{0} / \mathrm{G}_{1}, \mathrm{~S}$ and $\mathrm{G}_{2} / \mathrm{M}$ phases were quantified using FlowJo 7.0 software (FlowJo LLC).

SIRT2-associated pathway detection. The previous study indicated that SIRT2 was associated with the activity of the ERK1/2 signaling pathway in AML cells (16). Furthermore, it was previously shown that SIRT2 induced the migration and invasion of gastric cancer cells through the RAS/ERK/JNK/MMP-9 pathway (17). As a result, in order to investigate whether SIRT2 was associated with regulation of the RAS/ERK pathway in MM cells, the expression levels of HRAS, ERK and phosphorylated ERK (p-ERK) were assessed using western blot analysis and RT-qPCR in Sh-SIRT2 and Sh-NC cells after $48 \mathrm{~h}$ of incubation. 
A

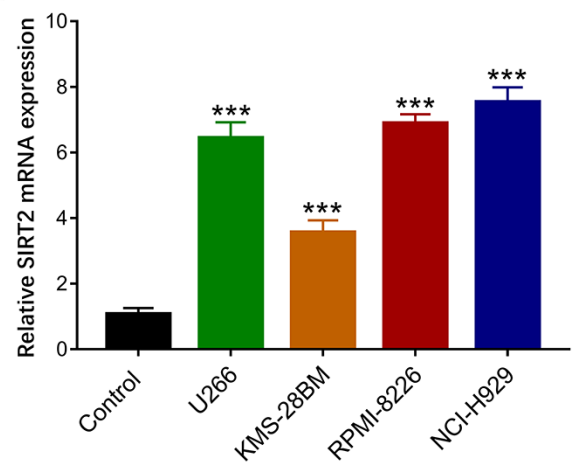

B

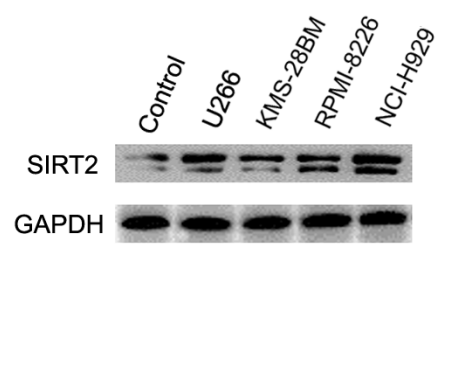

C



Figure 1. Detection of SIRT2 expression in MM cell lines and control cells. Comparison of relative (A) mRNA and (B and C) protein expression levels of SIRT2 between MM cell lines and control cells. ${ }^{*} \mathrm{P}<0.05,{ }^{* * *} \mathrm{P}<0.001$ vs. Control. SIRT2, sirtuin 2; MM, multiple myeloma.

Western blot analysis. Western blot analysis was performed as described previously (26). Briefly, the cells were lysed in RIPA lysis buffer (Sigma-Aldrich; Merck KGaA) and protein concentration was measured using a Pierce ${ }^{\mathrm{TM}}$ Rapid Gold BCA Protein assay kit (Thermo Fisher Scientific, Inc.). Subsequently, $20 \mu \mathrm{g}$ protein/lane was separated via $4-20 \%$ SDS-PAGE. The proteins were then transferred to nitrocellulose membranes (Qiagen $\mathrm{GmbH}$ ) and after blocking with 5\% BSA (Beyotime Institute of Biotechnology) at $37^{\circ} \mathrm{C}$ for $1.5 \mathrm{~h}$, the membranes were incubated with the following specific primary antibodies overnight at $4^{\circ} \mathrm{C}$ : Rabbit monoclonal anti-SIRT2 (1:2,000; Abcam; cat. no. ab211033), rabbit polyclonal anti-HRAS (1:2,000; ProteinTech Group, Inc.; cat. no. 15531-1-AP), rabbit monoclonal anti-ERK1/2 (1:10,000; Abcam; cat. no. ab109282), rabbit monoclonal anti-p-ERK1/2 (1:10,000; Abcam; cat. no. ab223500), rabbit polyclonal anti-PI3K (1:1,000; Abcam; cat. no. ab154598), rabbit polyclonal anti-p-PI3K (1:1,000; Abcam; cat. no. ab182651) and anti-GAPDH (1:10,000; Abcam; cat. no. ab245355). The following day, the membranes were incubated with goat anti-rabbit IgG H\&L (HRP) (1:20,000; Abcam; cat. no. ab97047) at $37^{\circ} \mathrm{C}$ for 1.5 h. The EasyBlot ECL kit (Sangon Biotech Co., Ltd.) was used for chemiluminescence detection. GAPDH was used as a loading control.

$R T$-qPCR. Total RNA was extracted from the cells using TRIzol ${ }^{\circledR}$ Reagent (Invitrogen; Thermo Fisher Scientific, Inc.) and was subsequently reverse transcribed into cDNA using the ReverTra Ace ${ }^{\circledR}$ qPCR RT Kit (Toyobo Life Science) at $42^{\circ} \mathrm{C}$ for 30 min. qPCR was performed using the TB Green ${ }^{\mathrm{TM}}$ Fast qPCR Mix (Takara Bio, Inc.). The following thermocycling conditions were used: Initial denaturation at $95^{\circ} \mathrm{C}$ for $30 \mathrm{sec}$; followed by 40 cycles of denaturation at $95^{\circ} \mathrm{C}$ for $5 \mathrm{sec}$, and annellation and extension, both at $61^{\circ} \mathrm{C}$ for a total of $15 \mathrm{sec}$. The experiments aimed to quantify SIRT2 and HRAS mRNA expression levels. The results were calculated using the $2^{-\Delta \Delta C q}$ method with GAPDH as the internal reference (27). The primer sequences used for RT-qPCR were as follows: SIRT2, forward 5'-ACGCTGTCGCAG AGTCAT-3', reverse 5'-CGCTCCAGGGTATCTATGTT-3'; HRAS, forward 5'-TGCCATCAACAACACCAAGTCTT-3', reverse 5'-CTGAGCCTGCCGAGATTCCA-3'; and GAPDH, forward 5'-GAAGGTGAAGGTCGGAGTC-3' and reverse 5'-GAAGATGGTGATGGGATTTC-3'.
Statistical analysis. All data are presented as the mean \pm standard deviation and all experimental studies were conducted in triplicate. The comparison between two groups was determined by unpaired Student's t-test and the comparison among multiple groups was determined by one-way ANOVA followed by the Dunnett-t test. All figure plotting and statistical analyses were performed using GraphPad Prism 7.01 (GraphPad Software, Inc.). $\mathrm{P}<0.05$ was considered to indicate a statistically significant difference.

\section{Results}

SIRT2 expression in MM cell lines and control cells. The relative mRNA expression levels of SIRT2 were increased in U266 $(\mathrm{P}<0.001)$, KMS-28BM $(\mathrm{P}<0.001), \mathrm{RPMI}-8226(\mathrm{P}<0.001)$ and NCI-H929 $(\mathrm{P}<0.001)$ cells compared with those in the control cells (Fig. 1A). In addition, western blot analysis indicated that the relative protein expression levels of SIRT2 were increased in U266, KMS-28BM, RPMI-8226 and NCI-H929 cell lines compared with those in the control cells (Fig. 1B and C). These data suggested that SIRT2 levels were increased in MM cell lines.

SIRT2 expression in patients with MM and healthy subjects. The relative mRNA expression levels of SIRT2 were higher in samples from patients with MM compared with those in samples from healthy donors $(\mathrm{P}<0.001$; Fig. S1).

SIRT2 expression following transfection of NCI-H929 and RPMI-8226 cells. As the increased expression of SIRT2 was the most significant in NCL-H929 and RPMI-8226 cells, these two cell lines were chosen for subsequent experiments. Post-transfection of MM cells with three shRNA-SIRT2 recombinant plasmids, the plasmid (shSIRT2 type 3) with the best knockdown effect on SIRT2 expression was selected for use in subsequent experiments (data not shown). The expression levels of SIRT2 mRNA (P<0.001; Fig. 2A) and protein (Fig. 2B and C) were decreased in the Sh-SIRT2 group compared with those in the Sh-NC group in NCI-H929 cells. In addition, the expression levels of SIRT2 mRNA ( $\mathrm{P}<0.001$; Fig. $2 \mathrm{C}$ and D) and protein (Fig. 2E and F) were reduced in the Sh-SIRT2 group compared with those in the Sh-NC group in RPMI-8226 cells. These data suggested that the transfection was successful. 
NCL-H929 cells

A

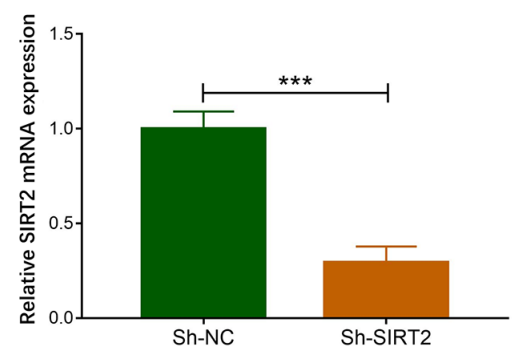

D

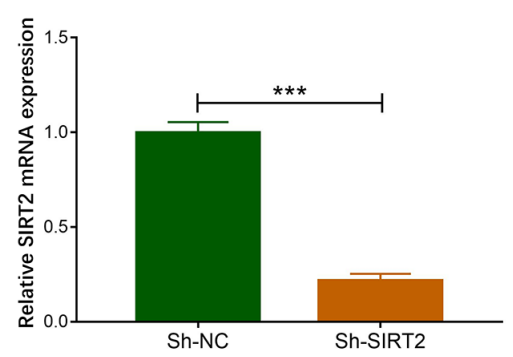

B

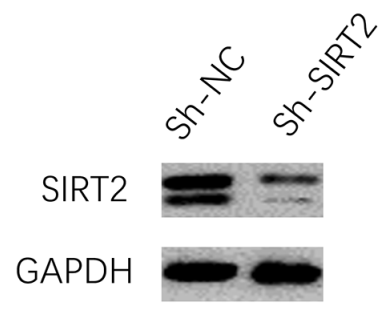

RPMI-8226 cells
C

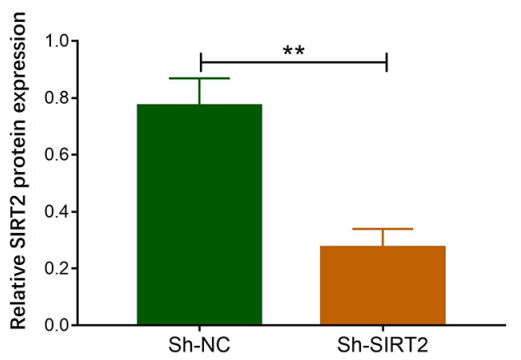

$\mathbf{F}$

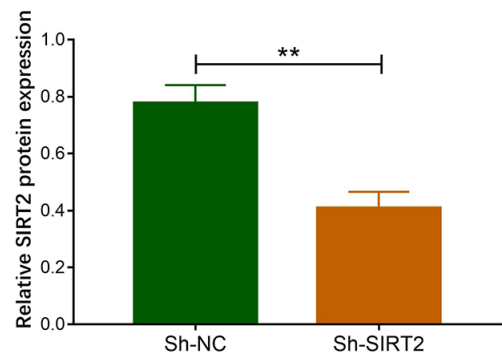

Figure 2. Detection of SIRT2 expression post-transfection. Comparison of (A) mRNA and (B and C) protein expression levels of SIRT2 between Sh-SIRT2 and Sh-NC groups in NCI-H929 cells. Comparison of (D) mRNA and (E and F) protein expression levels of SIRT2 between Sh-SIRT2 and Sh-NC groups in RPMI-8226 cells. ${ }^{* * *} \mathrm{P}<0.01{ }^{* * *} \mathrm{P}<0.001$. SIRT2, sirtuin 2; Sh-SIRT2, shRNA-SIRT2; Sh-NC, shRNA-negative control; shRNA, short hairpin RNA.

A

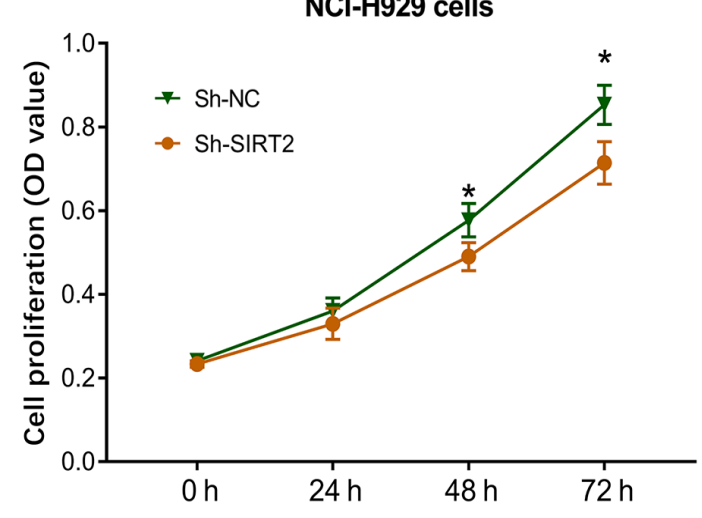

B

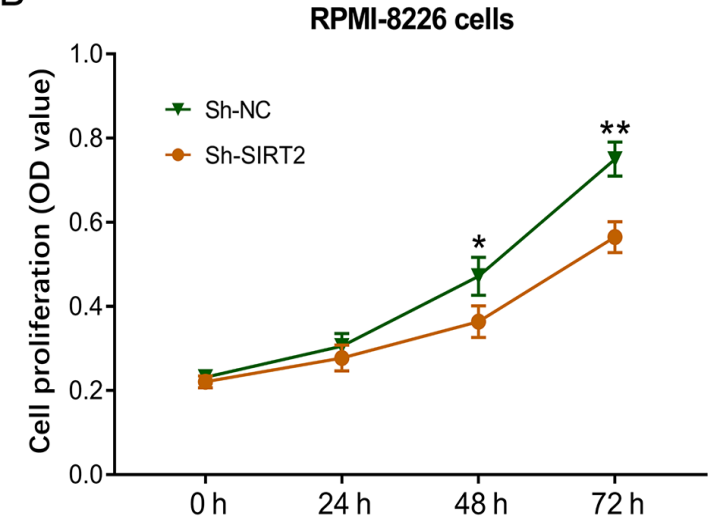

Figure 3. Proliferation of transfected NCI-H929 and RPMI-8226 cells. Comparison of cell proliferation between Sh-SIRT2 and Sh-NC groups post-transfection into (A) NCI-H929 and (B) RPMI-8226 cells. "P<0.05, ${ }^{* *} \mathrm{P}<0.01$ vs. Sh-SIRT2. SIRT2, sirtuin 2; Sh-SIRT2, shRNA-SIRT2; Sh-NC, shRNA-negative control; shRNA, short hairpin RNA; OD, optical density.

Effects of SIRT2 knockdown on the proliferation of NCI-H929 and RPMI-8226 cells. In NCI-H929 cells, cell proliferation was significantly decreased in the Sh-SIRT2 group compared with that in the Sh-NC group at 48 and $72 \mathrm{~h}$ post-transfection ( $\mathrm{P}<0.05$; Fig. 3A). In RPMI-8226 cells, cell proliferation was also reduced in the Sh-SIRT2 group compared with that in the Sh-NC group at $48(\mathrm{P}<0.05)$ and $72 \mathrm{~h}(\mathrm{P}<0.01)$ post-transfection (Fig. 3B). These data indicated that SIRT2 knockdown inhibited MM cell proliferation.

Effects of SIRT2 knockdown on apoptosis of NCI-H929 and RPMI-8226 cells. In NCI-H929 cells, the apoptotic rate was increased in the Sh-SIRT2 group compared with that in the Sh-NC group at $48 \mathrm{~h}$ post-transfection ( $\mathrm{P}<0.01$; Fig. $4 \mathrm{~A}$ and $\mathrm{B})$. Similar results were noted in RPMI-8226 cells; the apoptotic rate was significantly increased in the Sh-SIRT2 group compared with that in the Sh-NC group at $48 \mathrm{~h}$ post-transfection $(\mathrm{P}<0.01$; Fig. $4 \mathrm{C}$ and $\mathrm{D})$. These data suggested that SIRT2 knockdown promoted MM cell apoptosis.

Effects of SIRT2 knockdown on cell cycle progression in NCI-H929 and RPMI-8226 cells. In NCI-H929 cells, the percentage of the cells at $\mathrm{G}_{0} / \mathrm{G}_{1}$ phase was significantly increased $(\mathrm{P}<0.01)$, whereas the percentage of cells at $\mathrm{S}$ phase 
A

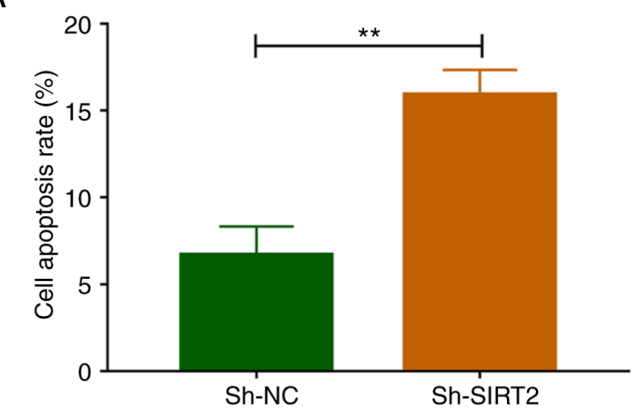

B

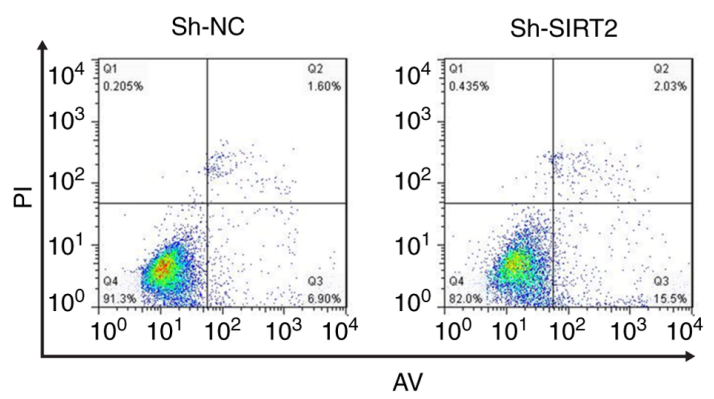

RPMI-8226 cells
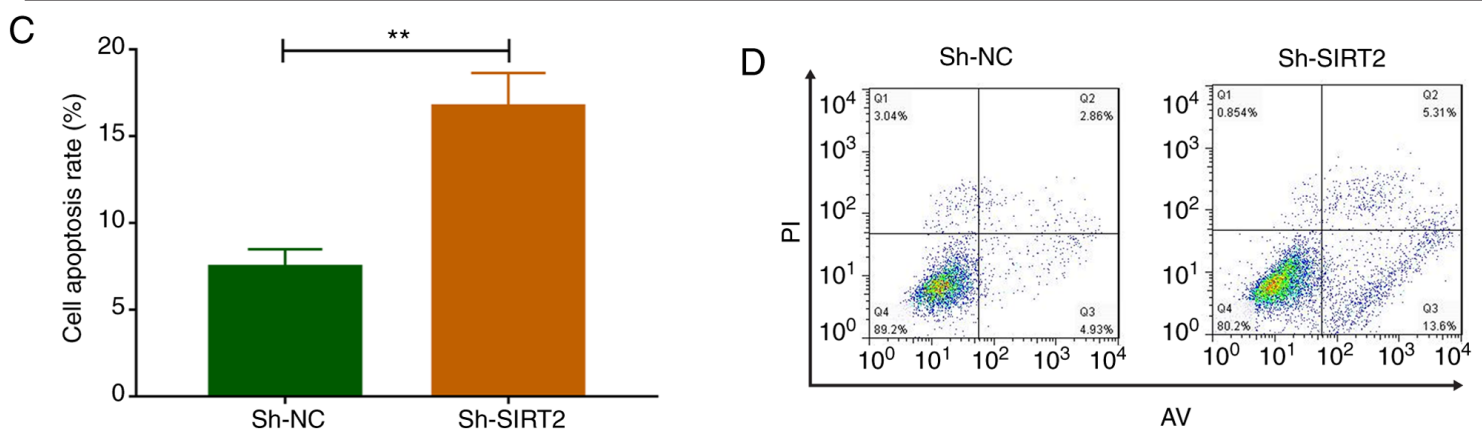

Figure 4. Apoptosis of transfected NCI-H929 and RPMI-8226 cells. Comparison of cell apoptosis between Sh-SIRT2 and Sh-NC groups following transfection into (A and B) NCI-H929 and (C and D) RPMI-8226 cells. ${ }^{* *}$ P<0.01. SIRT2, sirtuin 2; Sh-SIRT2, shRNA-SIRT2; Sh-NC, shRNA-negative control; shRNA, short hairpin RNA; PI, propidium iodide; AV, Annexin V.

$\mathrm{NCl}-\mathrm{H} 929$ cells
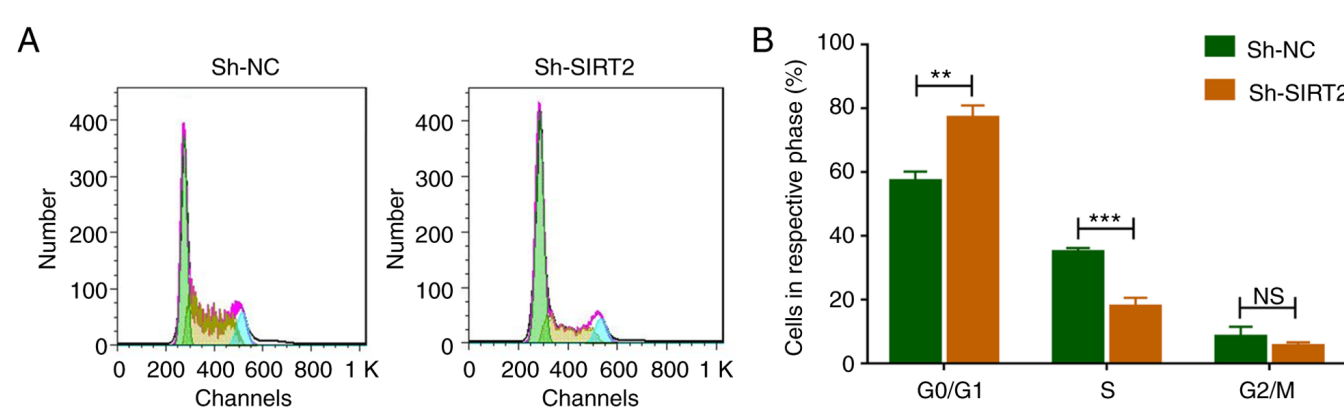

RPMI-8226 cells
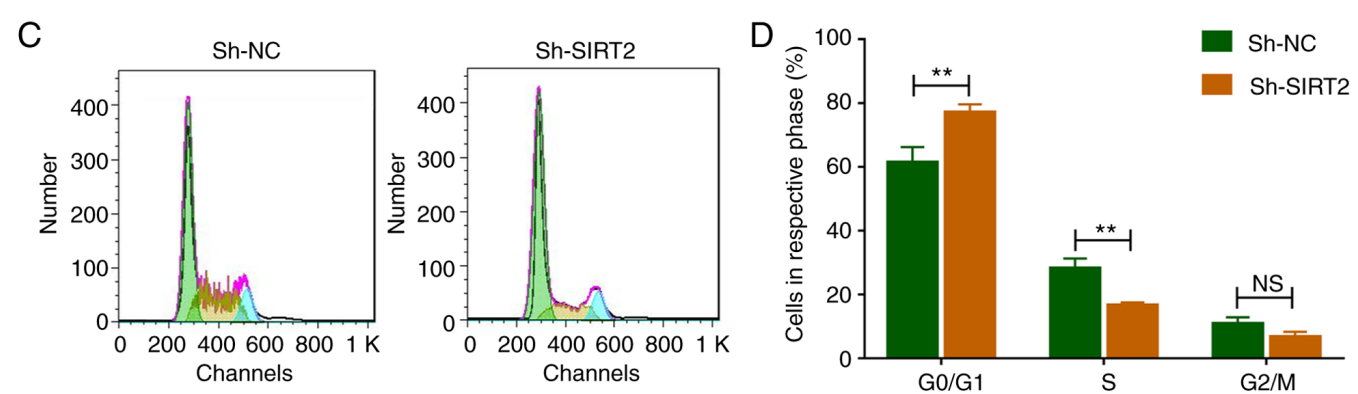

Figure 5. Cell cycle analysis of NCI-H929 and RPMI-8226 cells post-transfection. Comparison of cell percentages between the Sh-SIRT2 and Sh-NC groups following transfection into (A and B) NCI-H929 and (C and D) RPMI-8226 cells. The percentages of cells at $\mathrm{G}_{0} / \mathrm{G}_{1}, \mathrm{~S}$ and $\mathrm{G}_{2} / \mathrm{M}^{\mathrm{phases}}$ are shown. ${ }^{* *} \mathrm{P}<0.01$, ${ }^{* * * *} \mathrm{P}<0.001$. SIRT2, sirtuin 2; Sh-SIRT2, shRNA-SIRT2; Sh-NC, shRNA-negative control; shRNA, short hairpin RNA.

was significantly decreased $(\mathrm{P}<0.001)$ in the Sh-SIRT2 group compared with in the Sh-NC group (Fig. 5A and B). In RPMI-8226 cells, cell cycle arrest at $\mathrm{G}_{0} / \mathrm{G}_{1}$ arrest was also noted $(\mathrm{P}<0.01)$, which was accompanied by a reduction in the percentage of cells at $\mathrm{S}$ phase $(\mathrm{P}<0.01)$ in the Sh-SIRT group compared with in the Sh-NC group (Fig. 5C and D). These 
NCL-H929 cells

A

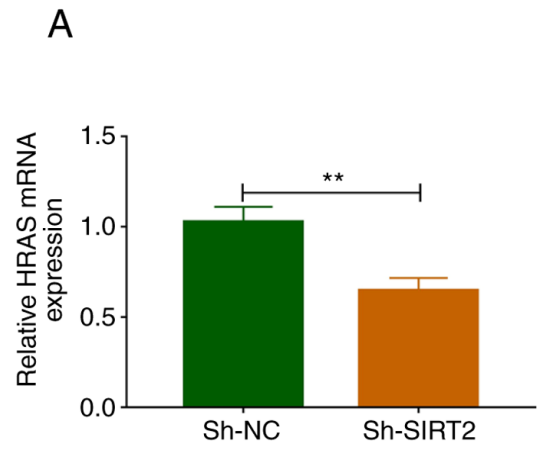

B

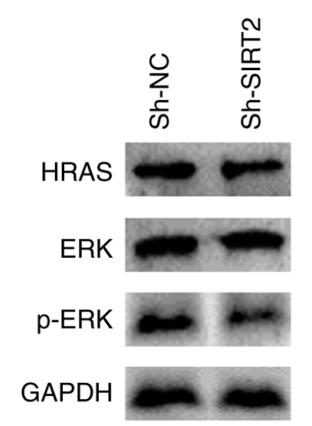

C

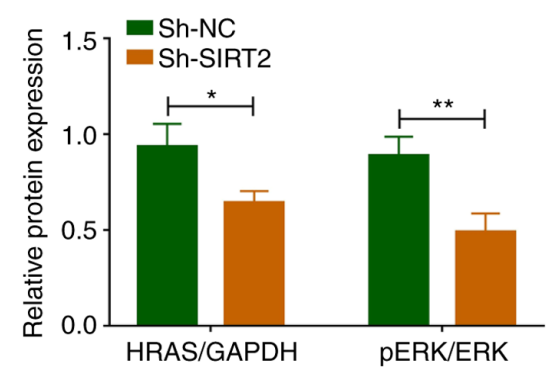

RPMI-8226 cells

D

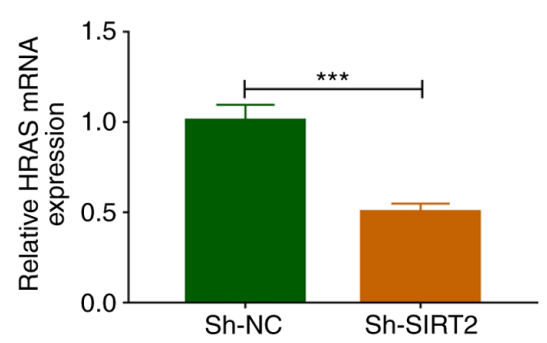

E

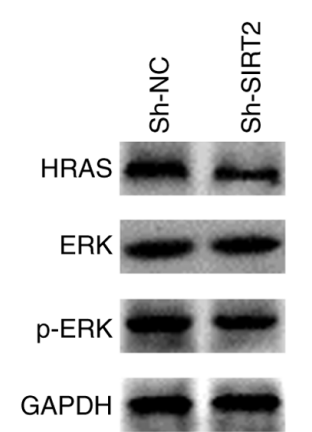

$\mathrm{F}$

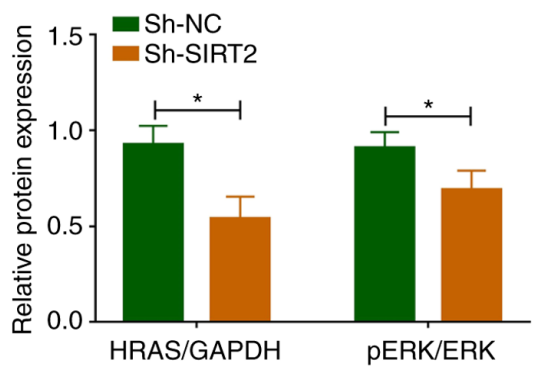

Figure 6. Effects of SIRT2 knockdown on RAS/ERK signaling. (A) Comparison of the mRNA expression levels of HRAS between the Sh-NC and Sh-SIRT2 groups in NCI-H929 cells. (B and C) Comparison of HRAS, ERK and p-ERK protein expression levels between Sh-NC and Sh-SIRT2 groups in NCI-H929 cells. (D) Comparison of the mRNA expression levels of HRAS between the Sh-NC and Sh-SIRT2 groups in RPMI-8226 cells. (E and F) Comparison of HRAS, ERK and p-ERK protein expression levels between the Sh-NC and Sh-SIRT2 groups in RPMI-8226 cells. ${ }^{\mathrm{P}}<0.05$, ${ }^{* *} \mathrm{P}<0.01$, ${ }^{* * *} \mathrm{P}<0.001$. SIRT2, sirtuin 2; Sh-SIRT2, shRNA-SIRT2; Sh-NC, shRNA-negative control; shRNA, short hairpin RNA; p-ERK, phosphorylated-ERK.

findings indicated that SIRT2 knockdown induced cell cycle arrest in MM cells.

Effects of SIRT2 knockdown on the RAS/ERK signaling pathway in NCI-H929 and RPMI-8226 cells. In NCI-H929 cells, the mRNA expression levels of HRAS were significantly decreased in the Sh-SIRT2 group compared with those in the Sh-NC group $(\mathrm{P}<0.01$; Fig. 6A). The protein expression levels of HRAS and p-ERK/ERK were also reduced in the Sh-SIRT2 group compared with those in the Sh-NC group (Fig. 6B and C). In RPMI-8226 cells, the mRNA expression levels of HRAS were significantly decreased in the Sh-SIRT2 group compared with those in the Sh-NC group $(\mathrm{P}<0.001$; Fig. 6D). The protein expression levels of HRAS and p-ERK were also reduced in the Sh-SIRT2 group compared with those in the Sh-NC group (Fig. 6E and F). In addition, in NCI-H929 (Fig. S2A and B) and RPMI-8226 (Fig. S2C and D) cells, the protein expression levels of $\mathrm{p}-\mathrm{PI} 3 \mathrm{~K} / \mathrm{PI} 3 \mathrm{~K}$ were lower in the Sh-SIRT2 group compared with those in the Sh-NC group. These findings suggested that SIRT2 knockdown inactivated the RAS/ERK signaling pathway in MM cells.

\section{Discussion}

In the present study, the data demonstrated that SIRT2 was highly expressed in U266, KMS-28BM, RPMI-8226 and NCI-H929 cell lines compared with those in normal plasma cells. Although SIRT2 knockdown inhibited cell proliferation, it promoted cell apoptosis and cell cycle arrest in MM cells. Furthermore, SIRT2 knockdown inactivated the RAS/ERK signaling pathway in MM cells.

SIRT2 is a $\mathrm{NAD}^{+}$-dependent deacetylase, which serves as a regulator of $\alpha$-tubulin acetylation, and is localized in the cytoplasmic and nuclear regions of the cell $(11,12)$. SIRT2 has been reported to serve an important role in regulating a variety of cellular physiological and biological processes. Notably, the implication of SIRT2 in the pathogenesis of malignancies, neurodegenerative diseases and inflammation-related diseases, has attracted increasing attention $(8,13,28,29)$. The involvement of SIRT2 in the pathological process of hematological malignancies has been confirmed by a previous study demonstrating that it was highly expressed in primary AML blasts compared with in hematopoietic progenitor cells from healthy donors. Moreover, its inhibition decreased cell proliferation and promoted apoptosis 
in AML via acetylation of AKT and further inactivation of $\beta$-catenin (18). In addition, another functional experiment (16) revealed that overexpression of SIRT2 led to increased multidrug resistance protein 1 expression, decreased drug accumulation and attenuated drug sensitivity. These effects were caused by activation of the ERK1/2 signaling pathway in AML cells and demonstrated the ability of SIRT1 to regulate the RAS/ERK/JNK/MMP-9 pathway, further promoting the development and progression of malignancies. Accumulating evidence has shown that activation of the RAS/ERK signaling cascade may result in FGFR3-mediated transformation, which is responsible for oncogenic cellular transformation in MM (19). According to these data, the current study examined whether SIRT2 was implicated in the pathological process of MM, which, to the best of our knowledge, has not been previously investigated. Initially, the expression levels of SIRT2 were compared between several human MM cell lines (KMS-28BM, U266, RPMI-8226 and NCI-H929) and normal plasma cells; the data demonstrated that SIRT2 expression levels were upregulated in MM cells compared with those in normal plasma cells. Furthermore, the data indicated that SIRT2 expression levels were higher in samples from patients with MM compared with those in samples from healthy donors.

The present study established the Sh-SIRT2 group by transfecting RPMI-8226 and NCI-H929 cells with a Sh-SIRT2 recombinant plasmid. These transfected MM cell lines aimed to explore the effects of SIRT2 knockdown on cell proliferation, apoptosis and regulation of the cell cycle. The data demonstrated that SIRT2 knockdown inhibited cell proliferation, whereas it promoted cell apoptosis and cell cycle arrest in MM cells. The possible reasons for these observations may include the following: i) According to previous evidence, SIRT2 expression may be positively associated with nicotinamide phosphoribosyltransferase (NAMPT) expression, and NAMPT knockdown has been shown to promote expression of the serine-threonine kinase glycogen synthase kinase $3 \beta$ by inducing AKT phosphorylation. This in turn may inactivate proto-oncogene $\beta$-catenin and suppress MM cell proliferation $(18,30)$. Therefore, SIRT2 knockdown may inhibit cell proliferation and cell cycle, while promoting apoptosis of MM cells. ii) Based on the previous studies reported, SIRT2 knockdown may inactivate the RAS/ERK signaling cascade (17), which could lead to suppression of FGFR3-mediated phenotypes $(16,17,19)$, further contributing to decreased proliferation, cell cycle arrest and increased apoptosis of MM cells.

SIRT2 has been reported to regulate the RAS/ERK signaling pathway in certain types of cancer $(16,17)$. For example, SIRT2 expression has been revealed to be positively associated with activation of the ERK1/2 signaling pathway in AML (16). An additional study revealed that the deacetylase activity of SIRT2 was inhibited by SirReal2 via the regulation of the RAS/ERK/JNK/MMP-9 signaling pathway $(16,17)$. Furthermore, interruption of RAS/ERK signaling promoted the expression of the checkpoint kinase 1 inhibitor, resulting in an increase in apoptosis and cell cycle arrest of hematopoietic malignant cells (31). Taken together, the aforementioned studies indicated that SIRT2 knockdown may decrease the expression levels of HRAS and p-ERK, which was also found in the current study, suggesting that this process could inactivate RAS/ERK signaling in MM. This may occur due to several possible reasons: i) SIRT2 knockdown may decrease PI3K expression; notably, a previous study indicated that PI3K interacts with RAS/ERK signaling (32). Therefore, reduction of PI3K expression may alleviate the stimulation of PI3K, leading to the inhibition of RAS/ERK signaling and a further decrease in the development of cancer (33). This speculation was verified by cellular experiments in which SIRT2 knockdown was shown to decrease PI3K expression in MM. ii) In addition, SIRT2 knockdown may decrease KRAS acetylation, which could further reduce activation of RAS downstream signaling markers, contributing to inhibition of RAS/ERK signaling (33). However, this hypothesis requires additional studies in order to be fully verified in MM. Although the present data demonstrated that SIRT2 knockdown inactivated RAS/ERK signaling in MM, its ability to inhibit MM cell malignant behavior via the RAS/ERK signaling pathway requires additional experiments. However, due to the limited budget, the relevant experiments were not performed, which is a limitation of the current study.

In conclusion, SIRT2 was revealed to be highly expressed in $\mathrm{MM}$ cell lines, and its knockdown inhibited cell proliferation, inactivated RAS/ERK signaling, and promoted cell apoptosis and cell cycle arrest in MM. Collectively, the present study implied that targeting SIRT2 may be a novel therapeutic option for the treatment of MM.

\section{Acknowledgements}

Not applicable.

\section{Funding}

This study was supported by the Health Research Project of Jing'an District of Shanghai (grant no. 2016MS10).

\section{Availability of data and materials}

The datasets used and/or analyzed during the current study are available from the corresponding author on reasonable request

\section{Authors' contributions}

JH conceived and designed the study. TD analyzed and interpreted data, and drafted the manuscript. TD and JH performed the experiments. Both authors participated in the writing and revision of the manuscript. JH supervised the project. JH and TD confirm the authenticity of all the raw data. Both authors read and approved the final manuscript.

\section{Ethics approval and consent to participate}

The protocol was approved by the Institutional Review Board of Shanghai Jing'an District Beizhan Hospital. All participants provided written informed consent for their participation in the study and for donation of bone marrow.

\section{Patient consent for publication}

Not applicable. 


\section{Competing interests}

The authors declare that they have no competing interests.

\section{References}

1. Brigle K and Rogers B: Pathobiology and diagnosis of multiple myeloma. Semin Oncol Nurs 33: 225-236, 2017.

2. Kazandjian D: Multiple myeloma epidemiology and survival: A unique malignancy. Semin Oncol 43: 676-681, 2016.

3. Rajkumar SV: Multiple myeloma: 2016 update on diagnosis, risk-stratification, and management. Am J Hematol 91: 719-734, 2016.

4. Nishida $\mathrm{H}$ and Yamada T: Monoclonal antibody therapies in multiple myeloma: A challenge to develop novel targets. J Oncol 2019: 6084012, 2019.

5. Isa R, Uoshima N, Takahashi R, Nakano-Akamatsu S, Kawata E, Kaneko H, Shimura K, Kamitsuji Y, Takimoto-Shimomura T, Mizutani S, et al: Sequential therapy of four cycles of bortezomib, melphalan, and prednisolone followed by continuous lenalidomide and dexamethasone for transplant-ineligible newly diagnosed multiple myeloma. Ann Hematol 99: 137-145, 2020.

6. Swyter S, Schiedel M, Monaldi D, Szunyogh S, Lehotzky A, Rumpf T, Ovádi J, Sippl W and Jung M: New chemical tools for probing activity and inhibition of the NAD(+)-dependent lysine deacylase sirtuin 2. Philos Trans R Soc Lond B Biol Sci 373: 20170083, 2018.

7. Liu G, Park SH, Imbesi M, Nathan WJ, Zou X, Zhu Y, Jiang H, Parisiadou L and Gius D: Loss of NAD-dependent protein deacetylase sirtuin-2 alters mitochondrial protein acetylation and dysregulates mitophagy. Antioxid Redox Signal 26: 849-863, 2017.

8. Patel VP and Chu CT: Decreased SIRT2 activity leads to altered microtubule dynamics in oxidatively-stressed neuronal cells: Implications for Parkinson's disease. Exp Neurol 257: 170-181, 2014

9. Szego EM, Gerhardt E and Outeiro TF: Sirtuin 2 enhances dopaminergic differentiation via the AKT/GSK-3 $\beta / \beta$-catenin pathway. Neurobiol Aging 56: 7-16, 2017.

10. Sunami Y, Araki M, Hironaka Y, Morishita S, Kobayashi M, Liew EL, Edahiro Y, Tsutsui M, Ohsaka A and Komatsu N: Inhibition of the NAD-dependent protein deacetylase SIRT2 induces granulocytic differentiation in human leukemia cells. PLoS One 8: e57633, 2013.

11. Jeong SG and Cho GW: The tubulin deacetylase sirtuin-2 regulates neuronal differentiation through the ERK/CREB signaling pathway. Biochem Biophys Res Commun 482: 182-187, 2017.

12. Nakagawa T and Guarente L: Sirtuins at a glance. J Cell Sci 124 833-838, 2011.

13. Kim HS, Vassilopoulos A, Wang RH, Lahusen T, Xiao Z, Xu X, Li C, Veenstra TD, Li B, Yu H, et al: SIRT2 maintains genome integrity and suppresses tumorigenesis through regulating APC/C activity. Cancer Cell 20: 487-499, 2011.

14. Luthi-Carter R, Taylor DM, Pallos J, Lambert E, Amore A, Parker A, Moffitt H, Smith DL, Runne H, Gokce O, et al: SIRT2 inhibition achieves neuroprotection by decreasing sterol biosynthesis. Proc Natl Acad Sci USA 107: 7927-7932, 2010

15. Funato K, Hayashi T, Echizen K, Negishi L, Shimizu N, Koyama-Nasu R, Nasu-Nishimura Y, Morishita Y, Tabar V, Todo T, et al: SIRT2-mediated inactivation of $\mathrm{p} 73$ is required for glioblastoma tumorigenicity. EMBO Rep 19: e45587, 2018.

16. Xu H, Li Y, Chen L, Wang C, Wang Q, Zhang H, Lin Y, Li Q and Pang T: SIRT2 mediates multidrug resistance in acute myelogenous leukemia cells via ERK1/2 signaling pathway. Int J Oncol 48: 613-623, 2016.

17. Li Y,Zhang M, Dorfman RG,Pan Y, Tang D, Xu L,Zhao Z, Zhou Q, Zhou L, Wang Y, et al: SIRT2 promotes the migration and invasion of gastric cancer through RAS/ERK/JNK/MMP-9 pathway by increasing PEPCK1-related metabolism. Neoplasia 20: 745-756, 2018.

18. Dan L, Klimenkova O, Klimiankou M, Klusman JH, van den Heuvel-Eibrink MM, Reinhardt D, Welte K and Skokowa J: The role of sirtuin 2 activation by nicotinamide phosphoribosyltransferase in the aberrant proliferation and survival of myeloid leukemia cells. Haematologica 97: 551-559, 2012.
19. Salazar L, Kashiwada T, Krejci P, Muchowski P, Donoghue D, Wilcox WR and Thompson LM: A novel interaction between fibroblast growth factor receptor 3 and the $\mathrm{p} 85$ subunit of phosphoinositide 3-kinase: Activation-dependent regulation of ERK by p85 in multiple myeloma cells. Hum Mol Genet 18: 1951-1961, 2009.

20. $\mathrm{Hu} \mathrm{J}$ and $\mathrm{Hu} \mathrm{WX}$ : Targeting signaling pathways in multiple myeloma: Pathogenesis and implication for treatments. Cancer Lett 414: 214-221, 2018

21. Moreau P, San Miguel J, Ludwig H, Schouten H, Mohty M, Dimopoulos M and Dreyling M; ESMO Guidelines Working Group: Multiple myeloma: ESMO Clinical Practice Guidelines for diagnosis, treatment and follow-up. Ann Oncol 24 (Suppl 6): vi133-vi 137, 2013.

22. Shaughnessy J Jr, Gabrea A, Qi Y, Brents L, Zhan F, Tian E, Sawyer J, Barlogie B, Bergsagel PL and Kuehl M: Cyclin D3 at $6 \mathrm{p} 21$ is dysregulated by recurrent chromosomal translocations to immunoglobulin loci in multiple myeloma. Blood 98: 217-223, 2001.

23. Draube A, Pfister R, Vockerodt M, Schuster S, Kube D, Diehl V and Tesch $\mathrm{H}$ : Immunomagnetic enrichment of CD138 positive cells from weakly infiltrated myeloma patients samples enables the determination of the tumor clone specific IgH rearrangement. Ann Hematol 80: 83-89, 2001.

24. Piracha ZZ, Kwon H, Saeed U, Kim J, Jung J, Chwae YJ, Park S, Shin HJ and Kim K: Sirtuin 2 isoform 1 enhances hepatitis B virus RNA transcription and DNA synthesis through the AKT/GSK-3 $\beta / \beta$-catenin signaling pathway. J Virol 92: e00955-18, 2018

25. Dryden SC, Nahhas FA, Nowak JE, Goustin AS and Tainsky MA: Role for human SIRT2 NAD-dependent deacetylase activity in control of mitotic exit in the cell cycle. Mol Cell Biol 23: 3173-3185, 2003

26. Nie H, Li Y, Wang C, Chen X, Liu B, Wu D and Ying W: SIRT2 plays a key role in both cell cycle regulation and cell survival of BV2 microglia. Int J Physiol Pathophysiol Pharmacol 6: 166-171, 2014.

27. Livak KJ and Schmittgen TD: Analysis of relative gene expression data using real-time quantitative PCR and the 2(-Delta Delta C(T)) method. Methods 25: 402-408, 2001.

28. Yang Y, Ding J, Gao ZG and Wang ZJ: A variant in SIRT2 gene 3'-UTR is associated with susceptibility to colorectal cancer. Oncotarget 8: 41021-41025, 2017.

29. Ma K, Lu N, Zou F and Meng FZ: Sirtuins as novel targets in the pathogenesis of airway inflammation in bronchial asthma. Eur J Pharmacol 865: 172670, 2019.

30. Zhang Y and Chi D: Overexpression of SIRT2 alleviates neuropathic pain and neuroinflammation through deacetylation of transcription factor nuclear Factor-Kappa B. Inflammation 41: 569-578, 2018.

31. Dai Y, Chen S, Pei XY, Almenara JA, Kramer LB, Venditti CA, Dent $\mathrm{P}$ and Grant $\mathrm{S}$ : Interruption of the Ras/MEK/ERK signaling cascade enhances Chk1 inhibitor-induced DNA damage in vitro and in vivo in human multiple myeloma cells. Blood 112: 2439-2449, 2008

32. Mendoza MC,Er EE and Blenis J: The Ras-ERK and PI3K-mTOR pathways: Cross-talk and compensation. Trends Biochem Sci 36: 320-328, 2011.

33. Song HY, Biancucci M, Kang HJ, O'Callaghan C, Park SH, Principe DR, Jiang H, Yan Y, Satchell KF, Raparia K, et al: SIRT2 deletion enhances KRAS-induced tumorigenesis in vivo by regulating K147 acetylation status. Oncotarget 7: 80336-80349, 2016.

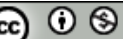

This work is licensed under a Creative Commons Attribution 4.0 International (CC BY-NC 4.0) License 